\title{
Taboo Trade-Offs, Relational Framing, and the Acceptability of Exchanges
}

\author{
A. Peter McGraw \\ University of Colorado \\ Philip E. Tetlock \\ University of California, Berkeley
}

\begin{abstract}
This article reports 4 experiments demonstrating the power of social-relational framing to complicate superficially straightforward economic exchanges of goods and services. Drawing from Alan Fiske's theoretical framework as well as Tetlock's sacred value protection model, the experiments demonstrate (a) pricing distortions and refusals to answer certain questions when people contemplate buying or selling objects endowed with special relational significance; (b) moral outrage and cognitive confusion when people are asked whether they would allow market-pricing norms to influence decisions that fall under the normative purview of communal-sharing, authority-ranking, and equality-matching relationships; and (c) elements of tactical flexibility in how people respond to breaches of relational boundaries (a willingness to turn a blind eye to taboo trade-offs when it is in their interest to do so). An agenda for future work is offered that explores how pragmatic, economic interests are balanced against the desire to be (or appear to be) the type of person who honors social-relational constraints on what should be considered fungible.
\end{abstract}

Many theories of judgment and choice rest on the premise that people make judgments and decisions as intuitive economists whose overriding goal in life is maximizing subjective expected utility (Gilovich, Griffin, \& Kahneman, 2002; Kagel \& Roth, 1995). People may not, of course, be perfect economists: They can fall prey to an assortment of well documented errors and biases. However, all the same, in both conceptual outlook and motivational orientation they are considered intuitive economists who are prepared to confront uncertainty, trade-offs, and opportunity costs to make the best decisions in competitive markets. One great advantage of adopting this framework has been a ready supply of elegant normative models for assessing judgmental biases and errors and for justifying attributions of irrationality.

Critics, however, have argued that the intuitive-economist framework is far too restrictive. They noted that ordinary people often resist the normative prescriptions of models anchored in that framework-even when those prescriptions are explicitly spelled out (Tetlock, 2003). If we want to understand why people often balk at the correct rational method of answering choice problems, we can adopt social-rela-

Requests for reprints should be sent to Peter McGraw, Leeds School of Business, 419 UCB, Boulder, CO 80309.E-mail: pmcgraw@ colorado.edu tional frameworks that focus on the identity-affirming and distancing functions that judgment and choice may serve (Tetlock, 2002). The logic of choice often may not be consequential: What is in this for me, and how can I get as much as possible? Rather, the logic of choice often may be that of role-constrained obligation: What kind of person do I claim to be in my relations to particular others, and what types of decisions would be compatible with this image of who I am (Beach, 1998; March \& Olson, 1989; Schlenker, 1985)?

\section{FISKE'S THEORY OF SOCIAL RELATIONS}

There are many possible social-relational frameworks that could guide work on consumer decision making (see, e.g., Parsons's, 1978, theory of pattern variables; Foa \& Foa's, 1974, resource theory; and Clark \& Mills's, 1979, communal vs. exchange theory). We rely, however, on Fiske's (1991, 1992) social-relational theory because it (a) provides an explicit and comprehensive taxonomy of the relational schemas that guide behavior; (b) is a useful starting point for identifying the sharp, qualitative boundaries that people place on the acceptability of certain forms of social cognition that should 
be unproblematic from an intuitive-economist perspective; and (c) has been extensively validated in both ethnographic and experimental research (Haslam, 2004).

Fiske's $(1991,1992)$ theory posited four types of relationships that people use to organize, evaluate, and coordinate most social interactions. These relationships and their rules serve as guiding principles or norms that can have a profound effect on behavior. The key defining features of each relational model are summarized here:

Communal sharing (CS) slices the social world into emotionally charged classes that allow us to differentiate in-groups and out-groups without degree of distinction. Everyone in a community-which could be as small as a romantic dyad or as large as a nation state-shares certain rights and incurs certain duties. Nonmembers may be excluded entirely. Within the relationships, people give as they can and take as they need.

Equality matching (EM) defines socially meaningful intervals that can be added or subtracted to keep score in social interaction. The social prototype is collegial or friendship networks in which in-kind or tit-for-tat reciprocity is a dominant exchange norm regulating the giving and taking of favors-here, it is critical to calibrate degrees of indebtedness and strive for balance.

Authority ranking (AR) imposes an ordinal ranking on the social world that permits lexical decision rules. One's location in this ranking scheme determines one's relative status in a collective and the prevailing direction of accountability for decision making. Military ranking serves as the social prototype: Majors must answer to colonels who, in turn, must answer to generals, with reversals only under exceptional circumstances.

Market pricing (MP) makes possible ratio comparisons of the values of diverse entities through the use of a single value or utility metric. This structure underlies capitalism and monetary transactions that range in sophistication from simple loans to financial instruments of such byzantine complexity that they baffle even Nobel laureates.

Within relational theory, there are no transitional forms. Each schema is a qualitatively distinct structure (Haslam \& Fiske, 1992). Although there is a strong tendency to use the same model across exchanges, the models may be used in conjunction with one another in interactions with the same person, and it is typical for a relationship to use multiple relational forms. For example, business partners may alternate driving each other to work, keeping with an EM relationship, and then divide up profits from their business venture in an MP manner, after which they celebrate at one or the other's home in a display of CM.
Relational schemas are too abstract to guide behavior without the aid of implementation rules that specify when, how, and to whom to apply each schema. Cultures solve this problem by providing a vast array of specific and often strict implementation rules. These rules should not be viewed as fixed or deterministic. Implementation rules evolve in response to challenges, and they are often ambiguous and underspecified at their contested margins. For example, in early 21 st century America, most people accept that there is a CS obligation to be compassionate toward, or at least refrain from harming fellow beings, but there continues to be ferocious debate over whether a spotted owl or a 4-month fetus should count as a fellow being meriting protection.

\section{TABOO TRADE-OFFS}

The capacity to make trade-offs efficiently is a defining attribute of homo economicus. From a relational perspective, however, people have a great deal more trouble with some types of trade-offs than others. Here it is useful to distinguish routine from taboo trade-offs. Routine tradeoffs entail comparisons between values that it is normatively acceptable to treat as fungible. We make such trade-offs on a daily basis (e.g., price-quality trade-offs at the supermarket). However, people are often extremely resistant to taboo trade-offs that extend MP fungibility norms into domains of life that people think of as having special, even sacred, status (Belk, Wallendorf, \& Sherry, 1989). The resistance cannot be fully explained by invoking the incommensurable objection that cognitive theorists often invoke to explain trade-off aversion: People lack pertinent precedents, and thus they do not know how much of $x$ to give up for $y$ when one of the dimensions quantifies a good or service that is unacceptable to sell in competitive markets. If an incommensurable objection per se were the obstacle, people would be merely confused by trade-offs that violate relational or normative boundaries and they would be hesitant and diffident in their answers. In fact, people are often morally outraged by the posing of such trade-offs, and many people refuse to respond to questions that they deem illegitimate, insulting, or absurd (see Mitchell \& Carson, 1989; Tetlock, Kristel, Elson, Green, \& Lerner, 2000; also see Medin, Schwartz, Blok, \& Birnbaum, 1999, for an illustration of moderating factors).

An explanatory framework proposed by Fiske and Tetlock (1997) for taboo trade-offs sheds light on these reactions (see also Baron \& Spranca, 1997). They defined a taboo trade-off as any explicit mental comparison or social transaction that violates deeply held intuitions about the integrity, even sanctity, of individual-to-individual or individual-to-society relationships and the values that animate those relationships. Building on Fiske's (1991) theory, Fiske and Tetlock proposed the following: 
People view trade-offs as impermissible and respond with varying degrees of indignation whenever the trade-offs require assessing the value of something governed by the socially meaningful relations and operations of one relational model in terms of a disparate relational model. (p. 256)

Stated differently, such trade-offs entail comparisons of the relative importance of secular values (e.g., money, time, and convenience) with sacred values that are supposed to be infinitely significant.

People are just not supposed to think or act in certain ways; as soon as one senses that others have committed a taboo trade-off, they become targets of moral outrage (as do those members of one's community who fail to censure them-i.e., there is a metanorm to enforce norms; see Fiske \& Tetlock, 1997). In one study, for example, Tetlock et al. (2000) either exposed people to routine tradeoffs, such as paying someone to clean their house or hiring a lawyer to defend them against criminal charges, or exposed them to taboo trade-offs, such as buying and selling adoption rights for orphans or buying and selling votes in elections for political offices. The authors found that, as expected, the latter elicited resistance, intense cognitive reactions (including punitive trait attributions to violators of taboos), emotional reactions (including expressions of anger, disgust, and contempt), and norm-enforcement reactions (including intentions to censure violators but also to censure those who fail to censure violators-metanorm enforcement). Exposure to taboo trade-offs was also morally contaminating; as a result, people tried to cleanse themselves morally. Those respondents who had thought that the decision maker had made the wrong choice in a taboo trade-off showed the greatest willingness to volunteer for a campaign to block a proposed ballot initiative that would legalize baby auctions.

In a study that investigated taboo trade-offs in a consumer context, McGraw, Tetlock, and Kristel (2003) examined the effect of applying an MP schema onto transactions involving objects that people received via the four fundamental relational modes. Respondents were asked their valuation of and willingness to sell the objects. Decisions were highly sensitive to the relational symbolism involved in the transaction. Although people accepted proposals to buy objects acquired in MP relationships as routine, the same proposals triggered progressively greater distress and erratically high dollar valuations as the goods received moved from AR and EM relationships to CS relationships. In short, people highly resisted applying the values from one relational schema (MP) to another (non-MP) schema.

\section{RELATIONAL NORMS AS GUIDES TO BEHAVIOR}

This article continues this exploration of the influence of social-relational norms on consumer behavior. Specifically, we explore people's reactions to taboo trade-offs and to the (mis)application of the normative rules of reciprocity within social exchanges. The reciprocity norm, typically associated with an EM schema, dictates that favors must be returned in rough proportion to their original size and generosity (Blau, 1964; Gouldner, 1962). But, as symbolic interactionists have long known (Stryker, 1980), EM relationships carry a lot more symbolic freight than just a compilation of quid pro quo understandings. Social identities are typically at stake. Participants make attributions in response to each move and countermove in exchange sequences: What kind of person would make an offer like that? What kind of person does the initiator of the offer apparently think I am to assume that I would think such an offer to be reasonable? What kind of person do I reveal myself to be by accepting or rejecting or even condemning such an offer?

We show that activating the norms linked to a particular relational schema can change the perceptions of and reactions to a proposed exchange. We also show how shifts in the framing of a relationship can profoundly influence the interpretation and perceived acceptability of an exchange (Aggarwal, 2004). According to the sacred value protection model (Tetlock, 2002), such relational framing should be especially effective when people seek ways to make trade-offs that, stated too baldly, would undermine claims to desired social identities. The model's reality constraint principle posits that, under such circumstances, people will search for ways to redefine morally corrosive taboo trade-offs as more benign and routine trade-offs.

\section{OVERVIEW}

The theme of this article can be stated succinctly: Marketing researchers make a mistake if they work exclusively within the framework of homo economicus. Social life is a delicate dance. Human beings are continually implicitly or explicitly negotiating the meanings of transactions and the nature of the social identities they would like to establish in the eyes of others. Each of our studies attempts to make this fundamental point in a different way.

Studies 1 and 2 examine the power of social-relational framing to moderate valuation decisions for possessions. Study 1 shows that trade-offs become taboo and pricing becomes severely distorted when the object to be sold symbolizes CS, EM, and AR relationships to significant others. Moreover, these effects are exacerbated when a would-be buyer makes a purchase offer with full knowledge of the relational significance of the object to its current owner. Study 2 reverses the process: It shows how the relationship of the buyer to the seller can moderate appropriate selling and buying prices of an object. Studies 3 and 4 focus on the effect of switching off or on a particular relational scheme: EM. These studies show-in interpersonal and political settings-that 
transactions that look acceptable within one relational framing quickly cease to be acceptable in another.

\section{STUDY 1}

The study replicates and extends the work of McGraw et al. (2003), manipulating the relational source of a possession through scenarios in which a person acquires an object via one of the four relationships in the Fiske model. To extend the work, we manipulate acquisition knowledge to test whether an overt failure to respect the social-relational significance of an object's origin increases the distress people experience when they contemplate a taboo trade-off. Observers were asked how the owner should react to an offer to buy the object from someone who either does or does not know the circumstances surrounding its original acquisition.

Dependent variables in the design included cognitive confusion, moral outrage, and prices recommended for seller and buyer. There was also an additional dependent variable that, in most circumstances, would be regarded as a nuisance but, in the current context, assumes considerable theoretical importance: namely, refusal to answer the question. Some respondents should feel that even the act of hypothetically assigning selling prices for taboo trade-offs casts doubt on the seller's moral integrity and social identity.

\section{Method}

Two hundred forty undergraduates at Ohio State University received partial course credit in exchange for their participation. Participants first examined a silver ballpoint pen (but were not told the retail price of \$5.00) and were then given a survey that described a graduate student who had received the pen within the context of a particular relationship. They were then asked to imagine that another student (i.e., the buyer) offered to purchase the pen. The second manipulation, acquisition knowledge, was introduced after the scenario. This fictitious buyer either knew or did not know the relationship context within which the graduate student had acquired the pen. Respondents were then asked to provide normative reactions to the offer as well as monetary assessments of the object's value.

Participants were randomly assigned to conditions in the 4 (relational source: CS, EM, AR, or MP) $\times 2$ (acquisition knowledge: buyer does or does not know the source of the object) between-subjects design.

The four scenarios are as follows:

CS: John is a graduate student here at Ohio State. When he first came here 2 years ago, he didn't know anyone. However, he soon became friends with the other people in his graduate research laboratory. The members of the lab are very considerate of one another. You could describe the atmosphere as family-like. Last summer, when John was studying abroad on a research grant, the people in his lab got together and sent him a care package. Included in it were some Lanterns [school newspapers], some snacks, and letters/notes from each of the people in the lab. Also included in this package was a pen from a conference that they all went to, but which John could not attend. Of course, all of these items were his to keep.

$A R$ : John is a graduate student here at Ohio State. When he first came here 2 years ago, he didn't know anyone. However, he soon became colleagues with the other people in his graduate research laboratory. All of his lab mates had the same faculty supervisor who provides leadership, a person whom they can go to as a mentor, role model, and authority figure. This advisor appointed John to be the head research assistant of the graduate students in the lab. John was given a Research Assistant Package from his advisor, which contained materials to help him do his job. One item that was John's to keep was a pen that, in its own way, marks the owner as an important member of the department.

EM: John is a graduate student here at Ohio State. When he first came here 2 years ago, he didn't know anyone. However, he soon became acquainted with the other people in the graduate research laboratory. They are easy to get along with and considerate of each other, and he quickly developed good working relationships with them. The graduate students frequently do things for each other to make graduate school as easy as possible. They are always helping one another out. For example, as a favor John helped another graduate student from the lab analyze data from an experiment. To return this favor, the other graduate student gave John this pen to keep.

MP: John is a graduate student here at Ohio State. When he first came here 2 years ago, he didn't know anyone. However, he soon became colleagues with the other people in his graduate research laboratory. As graduate students at Ohio State, they are often running out of office supplies due to their heavy academic load. There never seem to be enough supplies to go around. So, one of the graduate students often goes to a wholesale distributor to buy office supplies. When he's there, he buys more than he needs, and sells the rest to the members of the lab. This graduate student makes a little profit off the sale, but his lab mates (including John) find the prices reasonable and the arrangement convenient. John purchased this pen from this "salesman" graduate student.

Participants were asked to judge four statements designed to measure the appropriate response of the pen owner to the purchase offer. These statements were (a) John should reject the idea as completely inappropriate, (b) John should be happy to sell the pen at the right price, (c) John should be in- 
sulted by the offer to buy the pen, and (d) John should find the request strange or out of the ordinary. These items were designed to tap emotional and attitudinal reactions of distress, specifically feelings of confusion and outrage. Agreement with each statement was assessed on a 7-point category rating scale that ranged from 1 (completely disagree) to 7 (completely agree).

After completing the normative measures, respondents judged the appropriate price the pen owner should be willing to accept for the pen (WTA or selling price). They also estimated an appropriate price the buyer should offer (i.e., willing to purchase, WTP; or buying price). For both questions, participants were asked to justify their dollar valuation.

\section{Results}

To capture the overall affective and cognitive reactions of the pen owner, a composite variable-labeled henceforth as distress - was computed by averaging participants' agreement with the four normative statements (the second statement, "happy to sell at the right price," was reverse scored). The intercorrelations of agreement measures ranged from .22 to $.64(M=.46)$ and had acceptable reliability (Cronbach's $\alpha=$ .73). ${ }^{1}$ Higher values on the composite measure indicated greater distress with the request.

Figure 1 shows the average judged distress for conditions in the $4 \times 2$ (Relational Source $\times$ Acquisition Knowledge) design.

The main effects of relational source, $F(3,239)=33.1, \eta^{2}$ $=.30$, and knowledge of acquisition, $F(1,239)=16.7, \eta^{2}=$ .07 , were significant. ${ }^{2}$ An alpha level of .05 was used for all statistical tests. The analyses in all studies are significant at this level unless otherwise noted. Participants indicated the pen owner should experience significantly greater distress to the MP offer when the offer was made in non-MP relationship conditions $(M=4.5)$ than in the MP relationship condition $(M=2.8)$; planned contrast, $t(236)=9.4, d=1.5 .^{3}$ Distress was also more pronounced in the non-MP conditions when the purchaser was aware of the relational source $(M=$ 4.9 and $M=4.1$ for knowledge and no knowledge, respectively), $t(181)=4.8, d=.7$. This effect, however, is not present in the MP condition, where the means for knowledge and

${ }^{1}$ Removing any one of the variables from the composite measure reduces the reliability. This pattern occurs for the composite measures in the remaining studies unless otherwise noted.

${ }^{2}$ One measure of effect size reported throughout this manuscript is eta-squared $\left(\eta^{2}\right)$. It can be interpreted as proportion of variation explained or accounted for by the treatment manipulation. The index can range from 0 to 1, with larger numbers representing a larger effect. Keppel (1991) offered the following guidelines for interpreting effect size in behavioral research: .01 is a small effect, .06 is a medium effect, and $>.15$ is a large effect.

${ }^{3}$ Cohen's $d$ (Cohen, 1977) is provided for paired comparisons and contrasts as a measure of effect size. The statistic is calculated as the mean difference score divided by the standard deviation of difference scores. Cohen $d$ s of $0.2,0.5$, and 0.8 are interpreted as small, medium, and large effects, respectively.

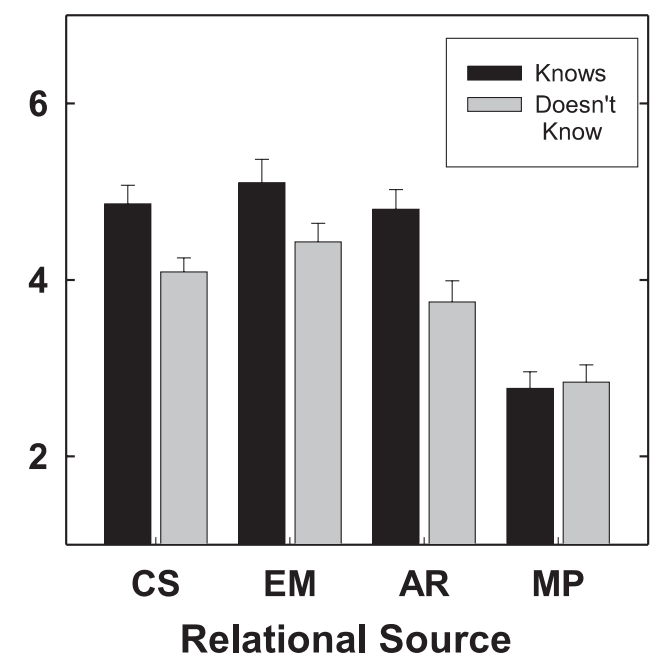

FIGURE 1 A composite measure of judged distress is plotted against relational context with separate bars for acquisition knowledge. Black bars and gray bars correspond to conditions when the would-be buyer knew or did not know how the pen was originally acquired. Error bars represent 1 standard error above the mean.

no knowledge were the same $(M=2.8)$. The Relational Frame $\times$ Knowledge interaction was not significant at the .05 level, $F(3,239)=2.5, p<.10, \eta^{2}=.03$, but the interaction was significant when the non-MP conditions were collapsed into a single variable, $F(1,239)=6.72, \eta^{2}=.03$.

Participants were asked the appropriate price the owner should be WTA (the selling price) for the pen and the appropriate price the would-be purchaser should be WTP (the buying price) for the pen. A substantial proportion of participants balked at providing dollar values for those questions. We employed two hypothesis-blind raters to judge selling and buying price refusals from the written justifications (interrater reliability was $94 \%$; disagreements were resolved through discussion).

Figure 2 shows the proportion of refusals by relational source. A substantial proportion of respondents refused to provide selling prices when the MP offer crossed relational boundaries (i.e., were applied to non-MP conditions). The effect of relationship was significant for both knowledge, $\chi^{2}(2$, $N=124)=17.6$, and no knowledge conditions, $\chi^{2}(2, N=116)$ $=21.2$. Refusal to provide selling prices did not differ significantly as a result of source knowledge, $\chi^{2}(2, N=240)=1.8$.

Overall, the effect on refusals was not as pronounced for buying as for selling prices; $17.5 \%$ of participants indicated that a purchase offer should not be made when the offer crossed relational boundaries. The effect, however, was significantly attenuated when the purchaser did not know how the pen was acquired, $\chi^{2}(2, N=180)=6.9$. Respondents felt that the purchaser should refrain from trying to buy an object originally acquired in a non-MP relationship, especially if the purchaser knew how the pen was acquired.

Were refusals related to the distress endorsed by respondents? We correlated refusals to provide dollar values with 


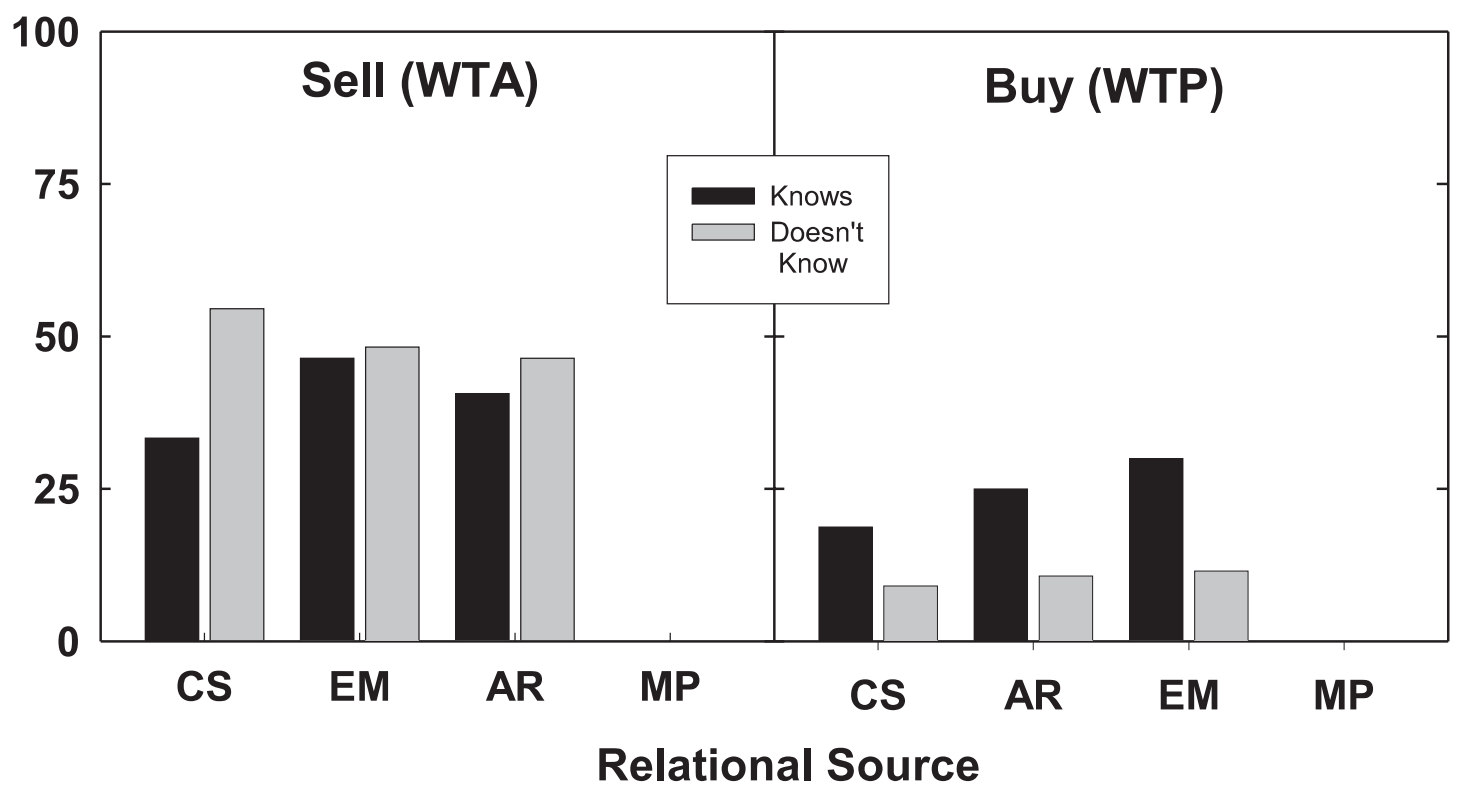

FIGURE 2 The percentage of respondents refusing to provide selling prices (left panel) or buying prices (right panel) are plotted against relational source with separate bars for acquisition knowledge conditions. Black bars and gray bars correspond to conditions when the would-be buyer knew or did not know, respectively, how the pen was originally acquired.

predicted distress for the non-MP conditions. There was a moderate correlation of distress with selling and buying price refusals ( $r=.30$ and $r=.33$, respectively). As judged distress increased, participants were more likely to refuse to provide selling and purchasing price. Refusals to provide dollar values also intercorrelated significantly for selling and buying questions $(r=.45)$.

Although refusals to provide dollar values provide strong support for our framework, it seriously undermines efforts to interpret the selling and buying price data. The refusals violate the assumption of source independence needed to conduct most parametric analyses. Moreover, the dollar values, WTA in particular, were highly skewed, with extreme values that reflected the volatility of dollar judgments (Kahneman, Schkade, \& Sunstein, 1998; McGraw et al., 2003).

A regression that predicted WTA from relational source and acquisition knowledge failed to reach significance, but when selling prices were transformed using log-values, which reduces the right skew in the data, a significant main effect of relational model was found, $F(3,142)=8.8, \eta^{2}=$ .16. A planned contrast between the non-MP conditions and MP was significant, $t(139)=4.8 ; d=1.0)$. Respondents said that more money should be demanded for the pen in the non-MP relationships. An effect of acquisition knowledge was not evident in selling prices. There were main effects of relationship, $F(3,181)=2.8 ; \eta^{2}=.05$, and knowledge, $F(1$, $181)=6.8, \eta^{2}=.04$, on log WTP values. ${ }^{4}$ Respondents indicated that the purchaser should offer more money when the pen was received from a non-MP relationship; planned contrast, $t(178)=2.6 ; d=.4$. Table 1 shows the mean and median values for the relational mode conditions.
Finally, we examined the ratio of selling price to buying price for individual participants collapsing across the knowledge condition. The ratios provide a measure of the relative discrepancy between selling and buying prices by putting individual responses on the same scale. Larger ratios indicate a greater discrepancy. The CS condition produced the greatest median ratio of WTA to WTP values (2.5:1), followed by EM and AR (for both, 2:1). MP showed no difference between buying and selling price (1:1).

\section{Discussion}

We found strong support for the explanatory usefulness of Fiske's taxonomy and Fiske and Tetlock's notion of taboo trade-offs. Participants reacted with greater distress to the MP offer when it crossed relational boundaries, and knowledge of acquisition source increased distress in these conditions. The object received in CS relationships was valued most highly, and the object received via MP relationships was valued least. EM and AR conditions fell in- between. In addition to reporting highly variable dollar valuations for EM, AR, and particularly CS objects, substantial percentages of respondents simply refused to participate in the mental exercise on moral or normative grounds (see Mitchell \& Carson, 1989, for an analogous phenomenon in the contingent-valuation literature). Refusals were also related to acquisition knowledge for buying prices. Participants felt that would-be buyers should respect the relational source of

${ }^{4}$ Results of the analysis are the same when conducted on the untransformed WTP values. 
TABLE 1

Mean and Median Values of Respondents' Selling and Buying Prices in Dollars by Relational

Conditions

\begin{tabular}{lrrrrrr}
\hline & \multicolumn{2}{c}{$M$} & & \multicolumn{2}{c}{$M d n$} \\
\cline { 2 - 3 } \cline { 6 - 7 } Relational Mode & \multicolumn{1}{c}{ WTA } & \multirow{2}{*}{ WTP } & & WTA & WTP \\
\hline Communal sharing & $2,500,109.45$ & 12.41 & & 30.00 & 5.00 \\
Equality matching & $34,548.31$ & 14.87 & & 10.00 & 8.00 \\
Authority ranking & 86.05 & 10.76 & & 20.00 & 8.00 \\
Market pricing & 5.65 & 5.56 & & 5.00 & 5.00 \\
\hline
\end{tabular}

Note. $\mathrm{WTA}=$ willing to accept $\mathrm{WTP}=$ willing to purchase. Data are shown collapsed across acquisition knowledge condition.

the object. Refusing to participate is socially awkward given the local norms of the experiment, but treating the request as unthinkable provides the surest way to affirm the commitment of the self to the broader norms of society and to shield one's social identity from the morally corrosive effects of taboo trade-offs (Tetlock et al., 2000). The resistance to the purchase offer was amplified by portraying the object as possessing special social-relational significance, symbolizing nontransferable and definitely nonfungible attachments to others (Belk, 1988; Csikszentmihalyi \& Rochberg-Halton, 1981; Grayson \& Shulman, 2000; Wallendorf \& Arnould, 1988).

One might wonder whether stronger support could have been obtained for an ordinal ranking of the relational modes in a repeated-measures design that required each participant to judge the relative offensiveness of MP intrusions into the $\mathrm{CS}, \mathrm{AR}$, and EM relational domains. The within-subject design, unlike the between-subjects design, gives respondents a shared context for comparison (Birnbaum, 1982; Hsee, Loewenstein, Blount, \& Bazerman, 1999). The repeated-measures design also allows us to ask whether the changes in buying to selling price ratios are greater than those obtained in between-subjects comparisons. Fiske and Tetlock's framework leads us to expect such a magnification of effects. Their framework treats schemas as usually unconscious cognitive operators that people become aware of only when repeated boundary disputes call competing relational logics to their attention. This is exactly what a repeated-measures design would do. If people are capable of making subtle normative distinctions in relational schemas, then they should be able to do so in a within-subjects design.

To explore this issue, we created an abbreviated within-subjects version of Study 1. Ninety-seven Ohio State undergraduates were given a survey that described a graduate student who had received a ballpoint pen valued at $\$ 5.00$. They read four single-sentence descriptions of the scenarios used in Study 1 and were told that another student offered to purchase the pen (acquisition knowledge was not mentioned). After each scenario description, participants completed three measures from Study 1. They responded to the normative-distress question tapping feelings of having been insulted, and they gave selling and buying prices.

An ordinal ranking of the relational modes emerged when people had the opportunity to gauge the relative offensiveness of MP intrusions into CS, AR, and EM relationships. There was a significant effect of condition on predicted feelings of insult, $F(3,279)=\mathrm{XX}, \eta^{2}=.48$. Feelings of insult were not significantly different from each other in the AR $(M$ $=4.66)$ and $\mathrm{EM}(M=4.4), t(93)=1.7, n s$, conditions, which fell significantly below responses in the CS condition $(M=$ 5.2 ), contrast $t(93)=5.9$. Finally, feelings of insult were lowest in the MP condition $(M=2.4), t(93)=68.5$. The percentage of respondents indicating that the pen should not be sold also followed the predicted pattern: $40 \%$ in CS, $24 \%$ in AR, $20 \%$ in EM, and $4 \%$ in MP. The same was true for refusals to provide buying prices: $23 \%$ in CS, $14 \%$ in $\mathrm{AR}, 12 \%$ in EM, and $2 \%$ in MP. The effect of social relationships on dollar ratios was stronger in this design. CS had the largest mean ratio in selling and buying prices (6.3:1), followed by EM (4.6:1), AR (2.3:1), and MP (1.2:1).

\section{STUDY 2}

Study 2 addressed the same issues in the context of the social relationship between buyer and seller, holding the source of the possession constant. Participants were asked to imagine that a would-be buyer approached them about an object that they had for sale. The buyer-seller relationship fit one of the four prototypical relationships within Fiske's framework. We then elicited minimum selling price (WTA), maximum buying price (WTP), and the desire to maximize profits from respondents. We expected the meaning associated with the relationship to influence the selling and buying prices of the object. But we expected a mirror image of the findings in Study 1 in which emotional resistance and unwillingness to sell peaked in the CS condition, followed by EM, AR, and then MP (where minimal affective ties were present; see also McGraw et al., 2003). We now expected the offered selling price to decrease as a function of the buyer's importance in one's life, bottoming out in the CS condition. It is socially awkward to do business in a profit-maximizing fashion across relational boundaries, and respondents should recognize this challenge and adjust their selling strategy accordingly.

\section{Method}

Undergraduates at Princeton University $(N=83)$ and University of Michigan $(N=69)$ participated in the study for partial course credit. ${ }^{5}$ They were presented with the following scenario:

Imagine that you were shopping for a new watch one day and saw one that you liked. You purchase the 
watch for $\$ 50.00$ and are happy with the deal you got. You tend to wear the watch a few days a week when you go to work or class. About a year after the purchase you receive a new watch as a birthday present. You like the gift watch better, so you decide to sell your current watch at a yard sale that your family is having.

Participants were randomly assigned to one of the four relational model conditions that manipulated the person inquiring about the watch that was for sale:

While you are at the yard sale a close family friend (CS), a neighbor with whom your family exchanges favors (EM), a teacher from high school (AR), or a customer (MP) notices the watch you are selling and says, "Wow, I really like that watch. It is exactly what I have been looking for. How much are you willing to sell it for?"

After reading the scenario, participants were asked the minimum amount of money they would sell the watch for (WTA). Then they were asked the maximum amount of money the person should offer for the watch (WTP). Finally, participants expressed their agreement with the statement, "I would try to maximize my profit from the sale of the watch," on a 9-point scale ranging from 1 (strongly disagree) to 9 (strongly agree).

\section{Results}

Respondents demanded the most money when a customer offered to purchase the watch (MP: $M=\$ 24.86$ ) and the least money when a close family friend offered to buy the watch (CS: $M=\$ 16.95)$. Values for the AR and EM relationships fell in-between; respondents wanted more from the former teacher (AR: $M=\$ 23.63$ ) than from the neighbor (EM: $M=$ $\$ 18.51)$. An analysis of variance confirmed the effect of the relational mode manipulation, $F(3,151)=2.8, \eta^{2}=.05$. Despite the medium effect size, the planned contrast between the non-MP conditions and the MP one was not significant at the .05 level, $t(148)=1.9, p<.10, d=.3$.

Participants felt that the buyer should offer more money (WTP: $M=\$ 36.94$ ) than the seller's minimum (WTA: $M=$ $\$ 20.96), t=13.2$. The ranking of the WTP values within the condition was the same as WTA values. The influence of relational source was again significant, $F(3,151)=4.9, \eta^{2}=$ .09 , although the differences in magnitude among the non-MP relationships were less extreme. Respondents indicated the close family friend should offer the least money (CS: $M=\$ 32.56$ ), followed by the neighbor (EM: $M=$ $\$ 34.19$ ) and then the teacher (AR: $M=\$ 35.63$ ). The customer was assigned the highest WTP for the watch (MP: $M=$

\footnotetext{
${ }^{5}$ There were no significant differences in the results between the groups. Thus, analyses were collapsed across the groups.
}

TABLE 2

Mean Values of Judged Willingness to Accept (WTA), Willingness to Pay (WTP), and Desire to Maximize Profits (Max) for Prototypical Relationships From Fiske's Relational Framework

\begin{tabular}{lccc}
\hline Relationship & WTA & WTP & Max \\
\hline Communal sharing & 16.95 & 32.56 & 3.2 \\
Equality matching & 18.51 & 34.19 & 3.9 \\
Authority ranking & 23.63 & 35.63 & 4.0 \\
Market pricing & 24.86 & 45.97 & 5.1 \\
Average & 20.96 & 36.94 & \\
\hline
\end{tabular}

\$45.97), which a planned contrast showed was significantly higher than the non-MP relationships, $t(148)=3.8, d=.6$.

Relationship condition was also a significant predictor of an intuitive economist's desire to maximize profits, $F(3,151)$ $=3.7, \eta^{2}=.07$. Respondents in the CS condition expressed the least agreement $(M=3.2)$ with a desire to maximize profits for the watch. The EM and AR condition followed $(M \mathrm{~s}=$ 3.9 and 4.0, respectively). Finally, the greatest desire to maximize occurred for the MP relationship ( $M=5.1)$, which fell above the midpoint of the scale (i.e., in the agree section of the scale). A planned contrast between the non-MP and MP conditions was significant, $t(148)=2.9, d=.6($ see Table 2$)$.

\section{Discussion}

People are sensitive to the meaning of relationships in their life and recognize that MP transactions need to be handled delicately. They understand that the amount of money offered for an item by a family friend, neighbor, or teacher differs from what a customer would offer. They also recognize that it is not acceptable to try to maximize profits when dealing with others who fall into the categories of CS, EM, or AR relationships. Moreover, people recognize that the offer they should expect from those meaningful others will fall below what would be expected in a purely MP exchange but above the value that they are willing to sell for, thus avoiding discomfort with the transaction.

We have shown that people are sensitive to the type of relationship they encounter in a consumer behavior context. The remaining studies further underscore how heavily people rely on the relational context to interpret the normative appropriateness of market and quasi-market transactions.

\section{STUDY 3}

We contrasted a typical EM exchange for household chores with conditions where respondents were asked to imagine a roommate either offers to pay them to do a household chore or offers to pay an additional share of a household bill (of the same amount) in exchange for doing the chore. The offer to pay the bill should make the offer more palatable because it 
can be more readily seen as part of an EM, turn-taking process. By contrast, asking a peer to take on a household chore is too uncomfortably close to the AR prototype of master-servant. The study serves as a test of the sacred-value protection model's reality constraint principle that predicts that people will seize upon the EM rationale as a means to avoid dealing with the encroachment of a MP structure on their relationship (Tetlock, 2002).

\section{Method}

Ninety-four undergraduates at Princeton University were paid for their participation in the study. Participants were randomly assigned to one of three between-subjects conditions. The first condition served as a baseline, where the roommate's request is consistent with the tit-for-tat nature (i.e., reciprocity norm) of an EM relationship.

Imagine that you recently moved into a new apartment with an acquaintance from school. You are still working out who will be responsible for particular chores. Suppose that your roommate tells you that he or she does not want to take out the garbage for personal reasons. He or she makes the following offer:

If you take out the garbage, he or she will always take care of vacuuming the apartment $(n=31 ; \mathrm{EM})$.

The second condition was designed to demonstrate the effect a MP proposal would have on reactions to the offer. It read:

If you take out the garbage, he or she will pay you $\$ 15.00$ a month for your effort $(n=30$; MP).

The third condition was designed to examine the reaction participants would have if the MP proposal were reframed in a form similar to an EM offer. It read:

If you take out the garbage, he or she will pay your share of the electric bill (which amounts to about $\$ 15.00$ a month) for your effort ( $n=33$; MP disguised).

Participants were then asked to judge via three statements the appropriate response to the request. These statements were (a) I would find the offer strange or out of the ordinary, (b) I would be happy with the offer, and (c) I would accept the offer. Respondents expressed their agreement on a 7-point scale ranging from 1 (completely disagree) to 7 (completely agree).

\section{Results}

We attempted to create a composite measure of distress from the three measures but found that the pattern of responses for the statement "would find the offer strange or out of the ordi- nary" differed from the remaining two measures. Thus, we labeled the variable confusion and analyzed it separately. There was a significant effect of condition, $F(2,93)=7.1, \eta^{2}$ $=.14$, on judgments. On average, participants indicated that they would not experience much confusion with the (typical) EM reciprocity offer $(M=3.1)$. However, in the MP condition, where an explicit payment is offered for doing the chore, participants expressed significantly more confusion than in the baseline group $(M=4.8), t(59)=3.5, d=.9$. Reactions to the disguised MP condition, however, were not different than the EM condition ( $M \mathrm{~s}=3.1$ and 3.3, respectively), $t(62)=.5, n s$. The MP condition, in fact, elicited more confusion than both the EM request and the disguised MP offer, contrast $t(91)=3.5, d=.9$.

The correlation between responses to the statements about happiness with the request and the willingness to accept the offer was .81 (Cronbach's $\alpha=.89$ ). Responses were averaged to create a variable labeled acceptability. There was a significant effect of condition, $F(2,93)=4.41, \eta^{2}=.09$, on the composite variable. Overall, participants were pleased with the offer, indicating relatively high levels of agreement with the statement. The highest level of agreement, however, occurred in the disguised MP condition. The mean level of agreement in the disguised MP condition $(M=6.1)$ reliably exceeded that of the $\operatorname{EM}(M=5.1)$ and MP $(M=4.9)$, contrast $t(91)=3.0, d=.7$, conditions. Keeping with the predictions of a strict utilitarian, participants felt that the amount of money offered was sufficient compensation, but the EM framing made the MP offer particularly appealing.

\section{Discussion}

By framing a MP request in terms of an EM exchange, the roommate could reduce the confusion and increase the acceptance of such a request. Participants were confused by the offer to pay them directly for the help (in effect, reducing them to the status of employees or even live-in servants). But people did not reject the same offer when it came in an acceptable EM, turn-taking package. This implies that the objection to the crassly overt MP offer was not grounded in the objection that the price was not right. The findings appear consistent with the sacred value protection model's reality constraint principle.

\section{STUDY 4}

Our final demonstration examined the effect of switching EM and MP relationships on and off on how people interpreted the Lincoln bedroom scandal that occurred near the midpoint of the Clinton presidency. We expected that the public distress over the large number of big campaign donors sleeping in the Lincoln bedroom (a vivid image of a sacred site subjected to secular contamination) would be high in a no-reason-given, pure-description condition but would be re- 
duced when politicians offer an EM rationale to explain the policy ("Friends do favors for friends") and would rise to new heights when politicians offer an MP explanation ("We computed the price that would raise the most money"). Building on work on the powerful tendency for people to seek out reasons that justify the judgments they make (Langer, Blank, \& Chanowitz, 1978; Shafir, Simonson, \& Tversky, 1993) as well as on work on motivated reasoning and ideological biases in reasoning (Kunda, 1999; Sniderman \& Tetlock, 1986), we also expected an effect of partisanship. Namely, we expected that anti-Clinton partisans would be less interested in reasons to become less irate (and, hence, less receptive to the EM excuse), whereas pro-Clinton partisans would be more eager to embrace the political-damage-minimizing, EM rationale.

\section{Method}

Seventy-seven undergraduate students at Ohio State University participated in the experiment for partial course credit. The study was run in 1997, soon after the Lincoln bedroom controversy became public knowledge. ${ }^{6}$ Twenty-seven participants who were randomly assigned to a control condition read an unadorned version of the Lincoln bedroom scenario:

The President of the United States, as everyone knows, lives in the White House. He has the authority to invite guests into this house for dinner or to stay overnight in the Lincoln bedroom. Many of the guests turn out to be large campaign contributors. Virtually all of the guests invited to stay in the Lincoln bedroom fall into this category ( $n=27$; unadorned).

Remaining participants were randomly assigned either to the MP version or the EM version of the scenario. The MP addendum to the unadorned scenario was as follows:

Imagine that we now discover from recently released documents how President Bill Clinton decided whom to invite for the honor of staying overnight in the Lincoln bedroom. The president's reasoning was as follows: Access to the White House is a scarce and valued resource, and we should send a message to potential campaign contributors that those who are willing to pay the price (i.e., $\$ 250,000$ or more) will be guaranteed invitations to stay in the Lincoln bedroom of the White House. There is nothing wrong with letting the economic laws of supply and demand determine who gets access to this resource $(n=24$; MP).

The EM addendum was as follows:

Imagine that we now discover from recently released documents how President Bill Clinton decided whom

\footnotetext{
${ }^{6}$ Informal debriefings found that more than half of the respondents were aware of the controversy.
}

to invite for the honor of staying overnight in the Lincoln bedroom. The president's reasoning was as follows: I think of big campaign contributors the same way I do of good friends, people who when times are rough back you up and back you up in a big way. I see nothing wrong with friends doing favors for friends and reciprocating those favors when it is possible to do so. That is how friendship and trust build: doing and returning favors over long periods of time $(n=26$; EM).

Before reading the scenarios, participants were asked, "What is your attitude toward President Bill Clinton?" and answered on a 7-point scale ranging from 1 (very negative) to 7 (very positive). After reading the scenario and addendum if present, participants responded to two measures of what they thought of the Presidential decision. The first measure ranged from 1 (immoral) to 7 (moral). The second measure ranged from 1 (admirable) to 7 (contemptible). Next, respondents answered the question, "Do you think voters should punish politicians who make decisions of this sort?" on a 7-point scale ranging from 1 (definitely no) to 7 (definitely yes). Finally, participants responded on two measures to the question, "How did reading this story make you feel?" The first measure ranged from 1 (no anger) to 7 (very angry) and the second measure ranged from 1 (no disgust) to 7 (very disgusted).

\section{Results}

Reactions to the Presidential decision were strongly correlated, ranging from .44 to $.77(M=.62$; the immoral measure was reversed scored). A composite variable that captures feelings of outrage and punitive intent was created from the five measures and had excellent reliability (Cronbach's $\alpha=.89$ ).

Three was a significant effect of condition on the composite variable, $F(2,74)=7.1, \eta^{2}=.14$. The unadorned facts elicited moderate levels of outrage and punitive intent $(M=$ 4.3). Reactions were more intense when the facts were accompanied by an explicit MP rationale $(M=4.9), t(74)=1.7$, $p<.10, d=.4$. However, reactions were attenuated $(M=3.8)$, $t(74)=1.8, p<.10, d=.6$, when the facts were accompanied by a rationale - the one, incidentally promoted by the White House- that invoked a friendship as opposed to economic norm, a rationale that affirmed the right of friends to do and return favors for friends. ${ }^{7}$ As a result, people's reactions to the MP and EM rationales were clearly different from one another ( $M=4.9$ and $M=3.8$, respectively), $t(74)=3.4, d=.9$.

There was also an effect of political partisanship on participants' responses to the outrage and punitive intent measures. The sample was roughly equally divided regarding their attitudes toward Bill Clinton (35\% were nonsupporters, $36 \%$ were supporters, and $29 \%$ were at the midpoint of the scale). A regression analysis, with the manipulated condition dummy coded, found that Clinton supporters responded with significantly less outrage than nonsupporters $(\beta=-.40, t=$ 


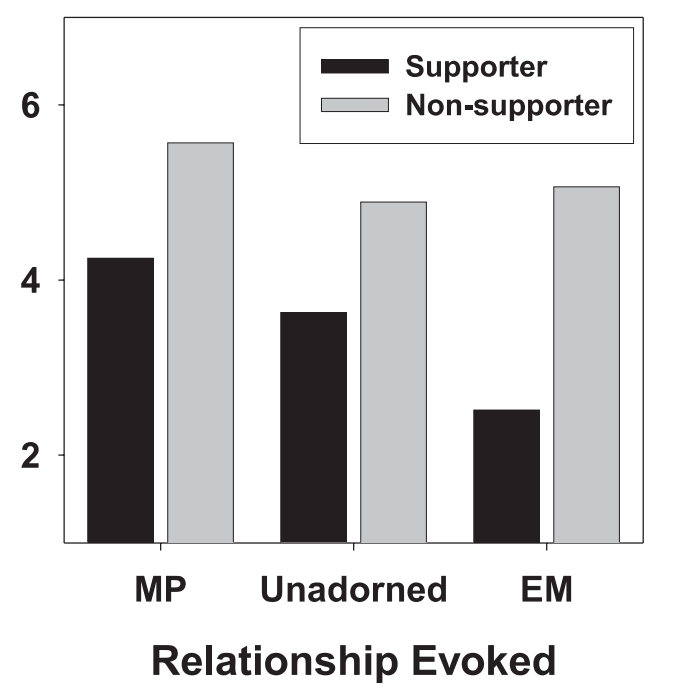

FIGURE 3 Predicted values of outrage and punitive intent are shown based on a regression equation with MP, unadorned version, and EM conditions dummy coded and crossed with attitudes toward Bill Clinton. Black bars (Clinton supporters) and gray bars (nonsupporters) show predicted values for respondents who were 1 standard deviation above and below mean attitude scores, respectively.

5.0). The relationship, however, was qualified by a Condition $\times$ Support interaction, whereby nonsupporters were relatively unaffected by the rationale $(\beta=-.02, t=0.2)$, and supporters were highly influenced by the rationale $(\beta=-.75, t=$ 3.8). Figure 3 shows predicted values of outrage and punitive intent based on the regression equation. In short, invoking the EM or reciprocity rule reduced outrage to politically tolerable levels for Clinton supporters.

\section{Discussion}

The study demonstrates that framing also matters in sharply contested political disputes. Relational reframing of prima facie suspect behavior can alter quite dramatically the interpretation of the deed, particularly among those disposed to think well of the political figure in question. The reciprocity norm central to EM-friends doing friends favors-reduces the taboo nature of events that many observers were initially inclined to interpret as MP contamination of a communally shared and sacred national site.

The introduction of the EM relational frame occurred after the initial disclosure of the apparent encroachment of secular value onto a sacred place. Given the reactions to the unadorned condition, it is safe to assume that Clinton supporters initially had a negative reaction, which was followed by the alleviation of their disgust. A question for follow-up work is whether the EM rationale served a genuine cleansing function (in which case, Clinton supporters would

\footnotetext{
${ }^{7}$ In both cases the contrasts were not significant at the .05 level. We suspect that this is due to low power associated with the small sample size in the study. Cohen's $d$ statistic indicated a medium effect size for both results.
}

feel no need to engage in compensatory conduct) or only a public relations function (in which case, people may feel some need to engage in such conduct).

\section{GENERAL DISCUSSION}

The first study showed that the monetary value that people place on possessions can depend on the interpersonal context within which people obtained the possessions and the meaning they now attach to those possessions. The results show that people draw a normative line in the sand between particularistic and universalistic relationships-that is, relationships in which people care very much about the identity of the actors from whom they receive goods and services and relationships in which people are indifferent to the identity of the actors, respectively (Foa \& Foa, 1974). People were far less willing to endorse the sale of a ballpoint pen if it was obtained in a CS, EM, or AR relationship than if it was obtained in a conventional MP manner. Moreover, substantial percentages of participants simply refused to consider the question of how much to sell the object for, treating the proposal as inappropriate and even insulting. Interestingly, people reported even more distress when they felt that the person making the purchase offer knew about the social or symbolic meaning of the object to the current owner. The social identity message appears to be: What kind of a person do you think would sell to a stranger an object that has personal meaning? Effects of relationships and source knowledge extended to perceptions of the prices that willing buyers should offer. Respecting the social-relational source is a two-way street in which buyers also need to acknowledge the symbolism inherent in the exchange that stems from the people originally exchanging the object.

The second study reverses the roles of buyers and sellers. It shows that our willingness to sell an object varies as a function of the identity of the person who wishes to purchase the object. Just as we demand more to sell an object that has CS, AR, or EM symbolic meaning, we demand less to sell an object of neutral significance when the would-be buyer has a CS, AR, or EM relationship to us. Again, would-be buyers are expected to be aware of and sensitive to the social-relational framing surrounding MP transactions.

Study 3 underscores the delicacy of EM relationships. There are some types of tasks that everyone is supposed to take their turn in performing. Citizens are not supposed to be able to buy their way out of obligations to serve in the military under draft laws. Housemates are not supposed to try to hire each other as servants, and those who violate this rule by asking each other to take on menial chores in return for monetary compensation do not understand the nature of the social contract. But how can we reconcile this argument with the neoclassical economic position that everything has a price and it is just a matter of discovering that price through bargaining? The study shows that housemates are more willing 
to sell their services to each other when they have a rhetorical cloak to disguise what they are doing. In the study, the cloak involves concealing the MP offer to buy services under the guise of picking up a share of a household bill that is supposed to be shared equally under EM norms. The "psycho-logic" is straightforward (although completely specious from an economic point of view): "You take my turn with the garbage take-out and I'll take yours paying the electricity bill."

The final study examines the tension between EM norms and MP standards in the context of a political scandal: the allegations that the Clinton presidency was explicitly selling off access to the Lincoln bedroom. Like Study 3, Study 4 shows that people are, on average, more forgiving when the transaction is framed (some might say "cloaked") as a matter of "friends doing favors for friends" but they balk when the transaction is framed as a matter of buying or selling access to an activity that is normally guided by CS norms. The study also shows something more. It shows that observers of the political scene play favorites, and this ideological divergence is especially pronounced when partisans are evaluating social identity-protective accounts for suspect behavior. Clinton supporters were more likely to accept the Clinton administration's justification that the president was simply reciprocating favors done by supporters and friends. Clinton detractors were more likely to assume the worst: that the unscrupulous Clinton administration allowed MP norms to intrude into a sphere where they do not belong.

\section{Taboo Markets}

There is widespread suspicion of the use of markets to allocate a fairly wide range of controversial goods and services (e.g., Sondak \& Tyler, 2004), a result that holds across individualistic and collectivist cultures (Shiller, Boycko, \& Korobov, 1991). E-bay recognizes this and prohibits the auctioning of Nazi memorabilia, letters or artwork from notorious murderers, and electric chairs as well as items that can give rise to legal difficulties such as the sale of human organs, sperm, or eggs. People are morally disgusted when such items are bought and sold like regular commodities (Rozin, Haidt, \& McCauley, 1993; Tetlock et al., 2000). Similarly, people often resist the application of MP practices to more complex markets such as giving incentives for adoptions (Landes \& Posner, 1978), trading pollution credits (Hahn, 1989), or creating futures markets for terrorist events (Weber \& Tetlock, 2003).

Sondak and Tyler (2004) found that people at least claim to be willing to sacrifice utility in the name of fairness, which may explain why markets that ignore issues of fairness may fail to clear (e.g., Kahneman, Knetsch, \& Thaler, 1986). Indeed, the overt application of secular values onto sacred entities can bring markets to a grinding halt. An implication of our studies is that people's resistance to such markets may be exaggerated by how they are framed. The more transparent a taboo market becomes, the greater the moral outrage and contamination it elicits. For example, a proposed model to give incentives for adoptions that would explicitly price White or male babies higher than African American or female babies would meet with much greater resistance than a proposal that would give vouchers to parents-to-be who were willing to adopt babies that were less likely to find a home (see Tetlock et al.'s, 2000, discussion of forbidden base rates).

\section{Culture, Ideology, and Implementation Rules}

Although marketing taboos can be identified across cultures, implicit in our analysis is the profound role that culture plays as a moderator of what people deem as taboo. Two or more entities are readily comparable only if people categorize them within the same relational domain. Implementation rules can, however, vary dramatically across time and place, so comparisons that are anathema in one culture can be routine in another. Fiske (1991) documented that, in 19th century West Africa, people deemed it perfectly respectable to calculate the exchange value in brass of a prospective slave or wife. It was also quite comprehensible, albeit a humiliating sign of how low one had sunk in the status system, to consider selling one's children into slavery in return for food. Conversely, it was - and in some pockets of rural Africa still is - bizarre to ask how many francs a person would accept for a millet field. Traditional West African society treated rights and persons as transferable in exchange for prestige goods but preserved farm land as communal. Fiske and Tetlock (1997) noted that, prior to the pressures of modernization and International Monetary Fund loan conditionalities that required extension of MP rules into agriculture, it made no more sense for West Africans to buy or sell the right to till the land than it did for Americans to buy or sell the right to breathe air or to vote. Of course, implementation rules change over time. What was at one time acceptable, such as paying to avoid conscription into the armed forces, is now considered anathema (Walzer, 1983). Ideology also has a moderating influence on the perceived appropriateness of transactions. Whereas liberals and conservatives find efforts to monetize babies, body parts, and basic rights and responsibilities of democratic citizenship abhorrent, we find that among libertarians the objections to these types of transactions wane (Tetlock et al., 2000). Moving left on the political spectrum toward socialism increases the tendency to find not only surrogate motherhood unacceptable but also the buying and selling of borderline controversial commodities such as medical care and legal representation as well as currently uncontroversial commodities such as houses and food. Devout egalitarians tend to see such exchanges as inherently inequitable because they put the poor at a profound disadvantage (and because they seem to carry the implication that the lives and rights of the poor are worth less than those who can pay large sums for doctors and lawyers). 


\section{Social Relationships and Marketing}

The results herein also have direct implications for advertising and marketing, some of which are already appreciated by practitioners. Large corporate actors often use the rhetoric of intimate relational models in their efforts to woo new customers and retain existing ones (Fournier, Dobscha, \& Mick, 1998; Johnson \& Selnes, 2004). Sometimes they promise to welcome you into a family (a CS metaphor) and sometimes into a partnership or friendship network (an EM metaphor). Sometimes they assume the pose of a wise, benevolent protector (typically an AR metaphor).

An intriguing set of questions for further work concerns the nature of the rhetorical strategies deployed and the effectiveness of these strategies. One possibility is that many people can indeed be persuaded, at some unconscious level, that they have symbolically entered into something other than a purely impersonal MP relationship with massive corporate entities. Indeed, the less cognitively sophisticated and more emotionally vulnerable people are, the more susceptible they may be to such appeals. As such, people may view brands or products in terms of a social relationship and behave as if the brand is a friend or partner (Fournier, 1998), even bestowing human characteristics onto the brand (McGill, 1998). Attachments of this sort may also help to explain a certain amount of otherwise irrational product loyalty. People do not want to disappoint corporate entities that they now see as the functional equivalent of friends and family. It is also possible, however, that these types of rhetorical manipulations backfire, striking people as just too transparently manipulative and disingenuous (Fournier et al., 1998). Even when these manipulations are successful, they may trigger a severe backlash if some mishap reveals the relational posturing to have been false (Aaker, Fournier, \& Brasel, 2004; Bechwati \& Morin, 2003; Koehler \& Gershoff, 2003). Consumers who have been gulled into thinking of themselves as part of a corporate family or partnership may feel especially bitter when they discover that the other party was treating them all along purely as objects of monetary calculation, even to the point of placing dollar values on their safety and lives.

Just as the exchange between two people can take on particular characteristics due to the nature of the relationship, the norms associated with brand relationships can influence people's evaluation of and behavior toward a brand. In related work, Aggarwal (2004) used Clark and Mills's (1979) distinction between communal and exchange relationships to examine the effect of relationship norm violations. For instance, a bank charging a fee for an additional service was seen as acceptable among those who viewed their relationship from an exchange (MP) perspective, but the same act elicited strong disapproval among those who viewed their relationship from a communal (sharing) perspective. Furthermore, Aggarwal found that the timing of a tit-for-tat request influenced perceptions of a business. When a communal relationship with a business was in place, respondents preferred a sufficient delay be- fore a return favor was requested, whereas the opposite occurred for an exchange relationship. People preferred to have a favor asked soon after they granted a favor. In short, if a brand's "behavior" violates the norms expected of the relationship, people experience distress and lower their evaluations of a brand, which in turn impel the person to reevaluate the special relationship status of the brand.

\section{CONCLUSION}

This article grapples-in a preliminary and exploratory fashion-with theoretical issues of foundational significance both to the field of consumer behavior and to the broader social sciences. Many economic theorists, as well as researchers in the domain of judgment and choice, have aggressively promoted the idea that virtually all human behavior can be captured within an MP framework in which each individual struggles as best he or she can to get the best possible deal within market constraints (Becker, 1991). The four experiments reported here show that, even in a culture often characterized as radically individualistic and capitalistic (early 21st century America), people often want to place sharp qualitative boundaries on the applicability of MP norms, viewing such norms as but one possible set of ways to judge the propriety of decisions within social settings. People's inherent sociability and the norms associated with their relationships can organize their exchanges.

Advocates of homo economicus can, of course, always argue that when the pricing pressure is sufficiently intense, normative inhibitions about treating goods and services as fungible will vanish into thin air. And there is evidence here-especially in Studies 3 and 4-that people are quite willing to tolerate MP encroachments into relationships governed by other norms when people have been given a rhetorical cloak to disguise what is happening. The tension between microeconomic and social-relational approaches should not be viewed as either-or. The challenge will be documenting the complicated situational and individual difference boundary conditions under which each approach provides explanatory leverage.

\section{ACKNOWLEDGMENTS}

Special thanks to Anna Cheung for valuable editorial and research assistance. We also thank Jason Riis for research assistance and Dan Bartles for helpful comments and suggestions.

\section{REFERENCES}

Aaker, J., Fournier, S., \& Brasel, A. (in press). When good brands do bad. Journal of Consumer Research.

Aggarwal, P. (in press). The effects or brand relationship norms on consumer attitudes and behavior. Journal of Consumer Research. 
Baron, J., \& Spranca, M. (1997). Protected values. Organizational Behavior and Human Decision Processes, 70, 1-16.

Beach, L. R. (1998). Image theory: Theoretical and empirical foundations. Mahwah, NJ: Lawrence Erlbaum Associates, Inc.

Bechwati, N. N., \& Morin, M. (2003). Outraged consumers: Getting even at the expense of getting a good deal. Journal of Consumer Psychology, 13, 440-453.

Becker, G. (1991). A treatise on the family. Cambridge, MA: Harvard University Press.

Belk, R. W. (1988). Possessions and the extended self. Journal of Consumer Research, 15, 139-168.

Belk, R. W., Wallendorf, M., \& Sherry, J. F. (1989). The sacred and the profane in consumer behavior: Theodicy on the odyssey. Journal of Consumer Research, 16, 1-38.

Birnbaum, M. H. (1982). Controversies in psychological measurement. In B. Wegener (Ed.), Social attitudes and psychophysical measurement (pp. 401-485). Hillsdale, NJ: Lawrence Erlbaum Associates, Inc.

Blau, P. (1964). Exchange and the power of social life. New York: Wiley.

Clark, M., \& Mills, J. (1979). Interpersonal attraction in exchange and communal relationships. Journal of Personality and Social Psychology, 37, $12-24$.

Csikszentmihalyi, M., \& Rochberg-Halton, E. (1981). The meaning of things: Domestic symbols and the self. Cambridge, England: Cambridge University Press.

Fiske, A. P. (1991). Structures of social life: The four elementary forms of social relations. New York: Free Press.

Fiske, A. P. (1992). The four elementary forms of sociality: Framework for a unified theory social relations. Psychological Review, 99, 689-723.

Fiske, A. P., \& Tetlock, P. (1997). Taboo trade-offs: Reactions to transactions that transgress the domain of relationships. Political Psychology, 18, 255-297.

Foa, U. G., \& Foa, E. B. (1974). Societal structures of the mind. Springfield, IL: Thomas.

Fournier, S. (1998). Consumers and their brands: Developing relationship theory in consumer research. Journal of Consumer Research, 24, 343-371.

Fournier, S., Dobscha, S., \& Mick, D. G. (1998). Preventing the premature death of relationship marketing. Harvard Business Review, 76, 42-51.

Gilovich, T., Griffin, D., \& Kahneman, D. (Eds.). (2002). Heuristics and biases: The psychology of intuitive judgment. New York: Cambridge University Press.

Gouldner, A. (1962). Anti-Minotaur: The myth of a value-free sociology. Social Problems, 9, 199-213.

Grayson, K., \& Shulman, D. (2000). Indexicality and the verification function of irreplaceable possessions: A semiotic analysis. Journal of Consumer Research, 27, 17-30.

Hahn, R. W. (1989). Economic prescriptions for environmental problems: How the patent followed the doctor's orders. Journal of Economic Perspectives, 3, 95-114.

Haslam, N. (2004). Relational models theory: A contemporary overview. Mahwah, NJ: Lawrence Erlbaum Associates, Inc.

Haslam, N., \& Fiske, A. P. (1992). Implicit relationship prototypes: Investigating five theories of the cognitive organization of social relations. Journal of Experimental Social Psychology, 28, 441-474.

Hsee, C. K., Loewenstein, G. F., Blount, S., \& Bazerman, M. H. (1999). Preference reversals between joint and separate evaluation of options: A review and theoretical analysis. Psychological Bulletin, 125, $576-590$.

Johnson, M., \& Selnes, F. (2004). Toward a dynamic theory of exchange relationships: Customer portfolio management. Journal of Marketing, 68, $1-17$.

Kagel, J. H., \& Roth, A. E. (Eds.). (1995). Handbook of experimental economics. Princeton, NJ: Princeton University Press.

Kahneman, D., Knestch, J. L., \& Thaler, R. (1986). Fairness as a constraint on profit seeking: Entitlements in the market. American Economic Review, 76, 728-741.
Kahneman, D., Schkade, D., \& Sunstein, C. R. (1998). Shared outrage and erratic awards: The psychology of punitive damages. Journal of Risk and Uncertainty, 16, 49-86.

Keppel, G. (1991). Design and analysis: A researcher's handbook (3rd ed.). Upper Saddle River, NJ: Prentice Hall.

Koehler, J. J., \& Gershoff, A. D. (2003). Betrayal aversion: When agents of protection become agents of harm. Organizational Behavioral and $\mathrm{Hu}$ man Decision Processes, 90, 244-261.

Kunda, Z. (1999). Social cognition: Making sense of people. Cambridge, MA: MIT Press.

Landes, E. M., \& Posner, R. A. (1978). The economics of the baby shortage. The Journal of Legal Studies, 7, 323-348.

Langer, E. J., Blank, A., \& Chanowitz, B. (1978). The mindlessness of ostensibly thoughtful action: The role of "placebic" information in interpersonal interaction. Journal of Personality and Social Psychology, 36, 635-642.

March, J., \& Olson, J. (1989). Rediscovering institutions: The organizational basis of politics. New York: Free Press.

McGill, A. (1998). Relative use of necessity and sufficiency information in causal judgments about natural categories. Journal of Personality and Social Psychology, 46, 357-364.

McGraw, A. P., Tetlock, P., \& Kristel, O. (2003). The limits of fungibility: Relational schemata and the value of things. Journal of Consumer Research, 30, 219-229.

Medin, D. L., Schwartz, H. C., Blok, S. V., \& Birnbaum, L. A. (1999). The semantic side of decision making. Psychonomic Bulletin and Review, 4, 562-569.

Mitchell, R. C., \& Carson, R. T. (1989). Using surveys to value public goods: The contingent valuation method. Baltimore: Johns Hopkins University Press.

Parsons, T. (1978). Action theory and the human condition. New York: Free Press.

Rozin, P., Haidt, J., \& McCauley, C. R. (1993). Disgust. In M. Lewis \& J. Haviland (Eds.), Handbook of emotions (pp. 575-594). New York: Guilford.

Schlenker, B. (1985). The self and social life. New York: McGraw-Hill.

Shafir, E., Simonson, I., \& Tversky, A. (1993). Reason-based choice. Cognition, 49, 11-36.

Shiller, R. J., Boycko, M., \& Korobov, V. (1991). Popular attitudes toward free markets: The Soviet Union and the United States compared. American Economic Review, 81, 385-400.

Sniderman, P. M., \& Tetlock, P. E. (1986). Symbolic racism: Problems of motive attribution in political analysis. Journal of Social Issues, 42, 129-150.

Sondak, H., \& Tyler, T. R. (2004). A relational perspective on the desirability of markets. Manuscript submitted for publication.

Stryker, S. (1980). Symbolic interactionism: A social structural version. Menlo Park, CA: Benjamin/Cummings.

Tetlock, P. E. (2002). Social-functionalist metaphors for judgment and choice: The intuitive politician, theologian, and prosecutor. Psychological Review, 109, 451-472.

Tetlock, P.E. (2003). Thinking about the unthinkable: Coping with secular encroachments on sacred values. Trends in Cognitive Science, 7, 320-324.

Tetlock, P. E., Kristel, O., Elson, B., Green, M., \& Lerner, J. (2000). The psychology of the unthinkable: Taboo trade-offs, forbidden base rates, and heretical counterfactuals. Journal of Personality and Social Psychology, $78,853-870$.

Wallendorf, M., \& Arnould, E. J. (1988). My favorite things: A cross-cultural inquiry into object attachment, possessiveness, and social linkage. Journal of Consumer Research, 14, 531-547.

Walzer, M. (1983). Spheres of justice. New York: Basic.

Weber, S., \& Tetlock, P. E. (2003, April 11). Futures trading and the internet [Editorial]. The New York Times, p. XX.

Received: March 17, 2004 